\title{
Auditory Evoked Responses and Computerized Tomography in Central Pontine Myelinolysis
}

\author{
R.S. YUFE, M.L. HYDE and K. TERBRUGGE
}

SUMMARY: We present the first case of central pontine myelinolysis (CPM) confirmed by high resolution computerized tomography $(C T)$ in which auditory brainstem responses $(A B R)$ revealed impaired conduction beyond the mid pons. The combined use of $C T$ and $A B R$ in the diagnosis of central pontine myelinolysis is discussed.

RESUME: Nous présentons le premier cas de myelinolyse pontique centrale confirmé par tomographie axiale à haute résolution $(C T)$. Chez ce patient les réponses auditives du tronc cérébral ( $A B R$ ) montrent une conduction atteinte passé la protubérance moyenne. L'emploi combiné de CTet ABR dans le diagnostic de cette entité est mis en lumière.

Reprint requests to Dr. Robert S. Yufe, $227-89$ Humber College Blvd., Rexdale, Ontario M9V 4B8 Canada.

From the Departments of Medicine. Etobicoke General Hospital; Otolaryngology, The Mount Sinai Hospital; and Radiology, Toronto Western Hospital.

\section{INTRODUCTION}

More than one hundred and ten cases of central pontine myelinolysis (CPM) have now been reported following the initial description of the disease by Mancall and Adams in 1959 (Burcar et al, 1977; Goebel and Zur, 1972; Messert et al, 1979).

Although previously thought to be exclusively a post mortem finding, increased recognition of the disease has lead to pre-mortem diagnosis in recent years (Boudin et al, 1963; Paguirigan and Letken, 1969).

Auditory evoked responses (ABR) and computerized tomography (CT) have independently been used to confirm the clinical diagnosis (Anderson et al, 1979; Stockard et al, 1976; Telfer and Miller, 1979; Wiederholt et al, 1977).

We describe another case of CPM in which both $A B R$ and $C T$ scan were confirmatory.

\section{Case Report}

A 49-year-old lady was brought to the emergency department with a three day history of anorexia, lethargy, increasing ataxia and frequent falls. She was known to consume large amounts of alcohol. Medications consisted of hydrochlorothiazide $50 \mathrm{mg}$. per day for hypertension and ampicillin for a recent urinary tract infection.

Physical examination revealed a drowsy, disoriented and confused lady who was rolling around aimlessly in bed. There were multiple ecchymoses over the extremities and orbits bilaterally. The temperature was $37.8^{\circ} \mathrm{C}$, blood pressure $160 / 110$ and pulse rate 80 per minute. The general physical examination was normal. Neurological examination revealed normal cranial nerves and fundi. She moved all four extremities. The reflexes were hypoactive, but both plantars were extensor. The hemoglobin was 15.9 grams per cent, white blood count 12,600 per cubic millimeter, prothrombin time 10.2 seconds, control of 10.8 seconds, partial thromboplastin time 31.7 seconds with a control of 36.2 seconds. Serum electrolytes revealed a sodium of 102 milliequivalents per litre, potassium 2.0 milliequivalents per litre, chloride less than 70 milliequivalents per litre, bicarbonate greater than 40 milliequivalents per litre, blood urea nitrogen 8 milligrams per decilitre and glucose 137 milligrams per decilitre. Blood and urine drug screen were negative. A skull $x$-ray was normal.

Treatment in the emergency room consisted of infusion of intravenous isotonic saline with potassium chloride, multivitamins and thiamine at a rate of 200 cc. per hour. After six hours there was little change in the clinical picture and her serum electrolytes were essentially unchanged. The rate of infusion of isotonic saline was decreased to $125 \mathrm{cc}$. per hour.

The following day she was improved. She was noted to be more alert and talkative with a serum sodium of 120 milliequivalents per litre, potassium 2.4 milliequivalents per litre, chloride 73 milliequivalents per litre, bicarbonate 35 milliequivalents per litre and blood urea nitrogen 4 milliequivalents per decilitre.

Four days following admission she was alert enough to demand to leave hospital. Her electrolytes and serum biochemistries were normal. The following day a psychiatrist found her to be staring vacantly into space with arms and legs in the fetal position and flexed. She was able to follow simple commands but speech consisted only of grunting. Her attention span was decreased and she was incontinent of urine.

Six days after admission, neurological consultation revealed an alert lady, but with no speech either spontaneously or to command. She responded appropriately to simple one-step commands but not sequences. The neck was supple. There were resolving hematomas over the right orbit and extremities. The pupils were symmetrical and reactive to light. The fundi were normal and she blinked to threat in both visual fields. Extraocular movements were full to command and on doll's head maneuvre. Corneal reflexes were present. The face was expressionless 
with weakness of both orbicularis oris muscles. The jaw jerk and facial reflexes were hyperactive. The gag reflex was reduced. Motor exam revealed a spastic quadriparesis. She was unable to stand without support. Limb reflexes were 4+ bilaterally with both plantars extensor. Ankle clonus was present bilaterally. She appeared to feel pin prick symmetrically in all four extremities and face. She was incontinent of urine and stool.

The following investigations were normal: Routine biochemical and hematological studies, liver function tests, thyroid indices, serum vitamin $\mathrm{B} 1 \dot{2}$ and folate, blood VDRL, 8:00 a.m. and 4:00 p.m. cortisols, liver and spleen scan, radionuclide brain scan and bilateral carotid arteriograms. Cerebrospinal fluid was clear and colorless with an opening pressure of 150 millimeters of mercury. The glucose was 70 milligrams per cent, protein 26 milligrams per cent, one red blood cell per cubic millimeter, one white blood cell per cubic millimeter, cultures, viral and fungal serology and CSF VDRL were negative. Immunoglobulin $G$ was 12 per cent of total protein.

An electroencephalogram revealed a moderate generalized disturbance in cerebral activity with superimposed bilaterally symmetrical and synchronous paroxysmal, high amplitude, irregular slow and sharp waves, suggestive of involvement of deep midline structures. A posterior fossa CT scan using the EMI model Mark One 160 by 160 matrix one week after admission was normal. Brainstem auditory evoked responses showed normal waves $\mathrm{I}, \mathrm{II}$, and III bilaterally with normal interwave latencies. However, only an ind istinct wave IV was obtained from the right ear and no response beyond wave III obtained from the left ear. (Fig. 1) A nonenhanced CT scan one month after admission using an AS \& E body scanner with a 520 by 520 matrix indicated a rounded fairly well defined area of decreased density within the pons. The lesion extended from the superior aspect of the brain stem to the cerebral peduncles. There was no significant mass effect or focal atrophy associated with the lesion. The enhanced CT scan showed no evidence of abnormal enhancement. The findings were thought to be compatible with a demyelinating process involving the pons. (Fig. II) Blink reflexes were consistent with a pontine lesion.

Two months following admission the patient requires nasogastric feedings. She remains quadriplegic and is unable to swallow. She is receiving intensive nursing care and physiotherapy.

\section{DISCUSSION}

The clinical picture of central pontine myelinolysis (CPM) consists of a rapidly progressive quadriparesis, pseudobulbar palsy, facial weakness, hyper-reflexia and extensor plantar responses. Most cases were described in alcoholics but electrolyte imbalance especially hyponatremia has been stressed as occurring in the majority of patients (Burcar et al, 1977). CPM has also been described in various other diseases including liver disease, Wilson's Disease, severe burns, amyloidosis and cerebral trauma. The association of CPM with Marchiafava-Bignami Disease has also be reported. (Ghatak et al, 1978).

The pathogenesis of the disease remains unknown and the susceptibility of the white matter of the basis pontis to the extensive demyelination unclear. Pathological studies have indicated relative sparing of axons within the demyelinated zone.

Messert et al (1979) have stressed the importance of iatrogenic precipitation of the disease by inappropriate rehy-
AUDITORY BRAINSTEM RESPONSES
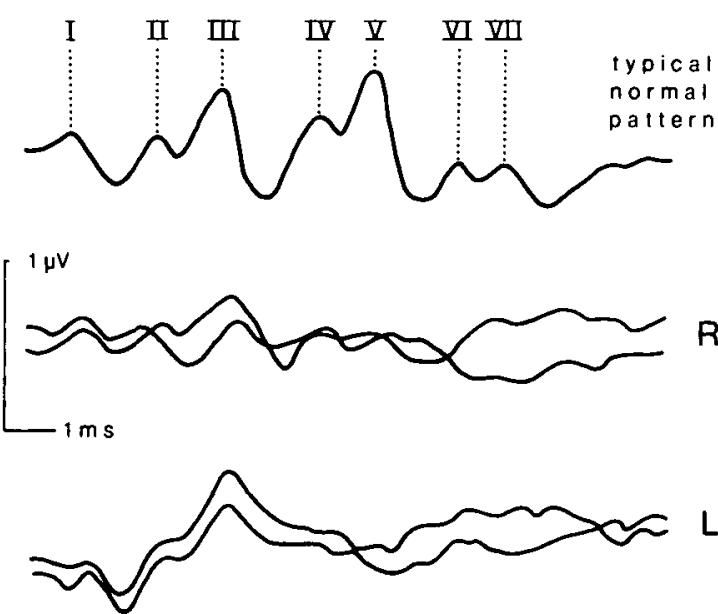

Figure $I$ - Normal auditory brainstem responses on top with comparison in central pontine myelinolysis below. The generator sites of these responses are as follows: I - acoustic nerve, II - cochlea nuclei, III - superior olivary complex, IV - lateral lemniscus, V - inferior colliculus, VI - medial geniculate body, VII - temporal lobe.

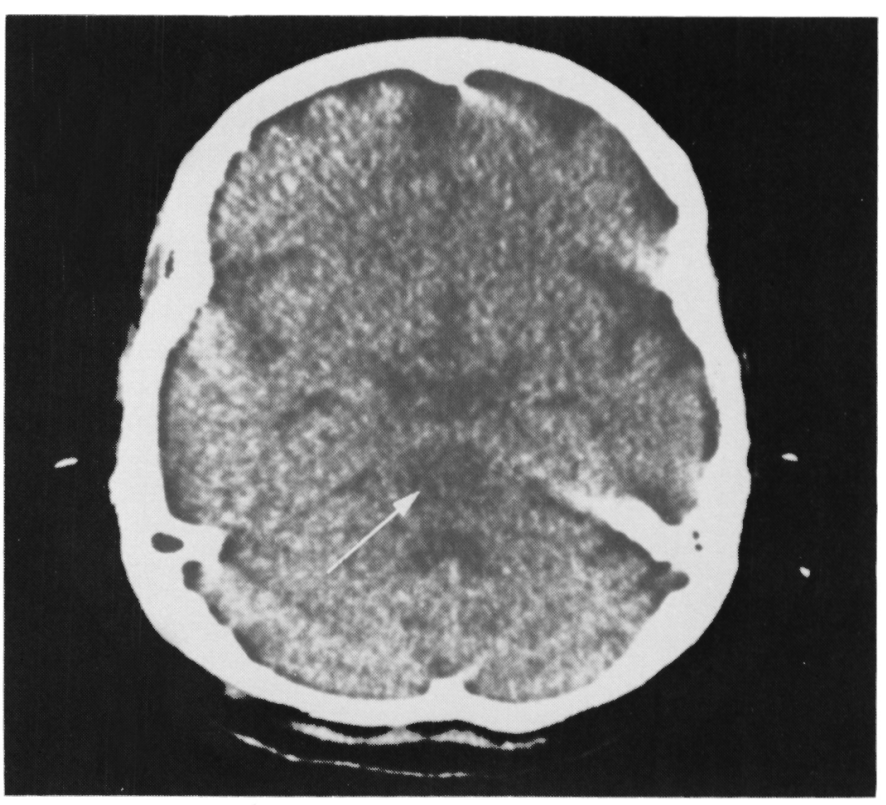

Figure 2 - Nonenhanced computerized tomographic scan at the level of the upper portion of the fourth ventricle shows a rounded area of decreased density within the superior aspect of the pons. (arrow) 
dration of at risk patients and indicated the need to rapidly correct hyponatremia yet restrict fluids. The mechanism of hyponatremia may be due to a combination of diuretics plus ethanol induced inappropriate antidiuretic hormone secretion.

Edema of the pons with strangulation of the myelin and perforating blood vessels by overvigorous use of intravenous fluids has been postulated to cause the extensive demyelination within the basis pontis (Poiners and McKeener, 1976). Such a mechanism may have been operable in our case.

ABR described initially by Jewett et al (1970) are reported to be useful clinically in the evaluation of patients with a host of neurological disorders (Ochs et al, 1979; Robinson and Rudge, 1977; Satya-Murti et al, 1978; Starr, 1976; Starr and Achor, 1975; Stockard and Rossiter, 1977; Tolosa and Zeese, 1979). In response to brief transient sounds such as clicks, a series of up to seven vertex positive waves can be obtained using surface electrodes on the human scalp, within the first ten milliseconds after each stimulus. These waves are generated by sequential activation of structures in the brainstem auditory pathways. Depth recordings, lesion experiments in cats and human clinical/pathological correlations suggest that waves I, II and III originate in the acoustic nerve, cochlear nuclei and superior olivary complex respectively. Waves IV and V are generated within the lateral lemniscus and inferior colliculus respectively. The generator sites for waves VI and VII are still in dispute and thought to arise within the medial geniculate body and temporal lobe respectively (Buchwald and Huang, 1975; Janett, 1970). Waves VI and VII are not always present in normals and are of little clinical value at the present time (Chiappa et al, 1979).

In Figure 1 are shown a typical normal ABR wave series, and the results obtained in this patient. Responses were measured using a Madsen ERA 74 system. Electrodes were on the forehead in the midline at the hairline, and on the two mastoids. System bandpass was $100 \mathrm{~Hz}$ to 3000 Hz. Stimuli were 83 microsecond rectangular pulses, delivered monotically at 10 per second and $90 \mathrm{~dB} \mathrm{nHL}$ via
TDH 39 headphones. The contralateral ear was masked with $50 \mathrm{~dB}$ white noise. Using the mastoid electrode on the stimulated ear as reference, two replicate ABRs to 4000 stimuli were obtained on each ear.

The patient's hearing thresholds could not be assessed conventionally, but contralateral acoustic reflexes were obtained at $1 \mathrm{kHz}$ and $2 \mathrm{kHz}$ in both ears. The reflex was absent at 4 $\mathrm{kHz}$ in the right ear - not an uncommon finding in purely cochlear disease. In the ABR records of Figure 1, a clear wave I can be seen bilaterally. This suggests that neither cochlea was sufficiently damaged to prevent effective excitation of the brainstem pathways. Thus, any subsequent abnormality of the ABR series can be ascribed to brainstem disease rather than to peripheral auditory dysfunction.

On the left side, waves I, II and III are clear and normal. There are no subsequent waves, suggesting disruption of neural conduction above the superior olive, but below the lateral lemniscal nuclei.

On the right side, waves I, II and III are also normal, and there is a vestigial wave IV, suggesting partial involvement of the lemniscal nuclei on that side. There is no recordable activity from the inferior colliculi, since wave $\mathrm{V}$ is absent bilaterally. Stockard et al (1976) and Wiederholt et al (1977) have described two cases in which CPM was suspected clinically and ABRs were normal, showing prolonged latency intervals between waves $I$ and V. CT scans were not performed in either of these cases.

In our case it appears that brainstem involvement is more severe or extensive, since wave $\mathrm{V}$ was completely obliterated.

Recently, two additional cases of CPM were described with the diagnosis confirmed on CT scan (Anderson et al, 1979; Telfer and Miller, 1979). Both cases in addition to our own showed a large radiolucent lesion in the mid portion of the pons that did not enhance on intravenous infusion of contrast material. The case described by Telfer and Miller (1979) and our case failed to show the lesion using older CT equipment. Higher resolution scanners are thus important in detecting the lesion of CPM.
We wish to emphasize the following:

1. Central pontine myelinolysis is a clinical diagnosis that can be confirmed by the use of combined $A B R$ and high resolution $C T$ scan.

2. The mechanism of pontine demyelination remains unclear. Electrolytes should be rapidly corrected. Excessive fluid replacement should be avoided to hopefully prevent the disease.

3. Our patient remains severely involved neurologically, but clinical recovery can occur. Vigorous support and treatment of the intercurrent illness is thus important. Serial ABR measurement may be useful in following the course of the disease.

\section{ACKNOWLEDGEMENT}

I would like to thank Miss Janet Rawski for her patience and typing of the manuscript.

\section{REFERENCES}

ADAMS, R.D., VICTOR, M., MANCALL, E.L. (1959). Central Pontine Myelinolysis. Arch. Neurol. Psychiatry 81: 154-172.

ANDERSON, T.L., MOORE, R.A., GRINNELL V.S., et al (1979). Computerized Tomography in Central Pontine Myelinolysis. Neurology (Minneap.) 29: 1527-1530.

BOUDIN, G.l., LABET, R., LYON G. et al (1963). La myélinolyse centrale de la protuberance. Presse Med. 7I: 2080-2082.

BUCHWALD, J.S., HUANG, C.M. (1975). Far Field Acoustic Response: Origins in the Cat. Science. 187: 302-304.

BURCAR, P.J., NORENBURG. M.D., YARNELL, P.R. (1977). Hyponatremia and Central Pontine Myelinolysis. Neurology (Minneap.) 27: 223-226.

CHIAPPA, K.H., GLADSTONE, K.J., YOUNG, R.R. (1979). Brainstem Auditory Evoked Responses: Studies of Waveform Variations in Fifty Normal Human Subjects. Arch. Neurol. 36: 81-87.

GHATAK, N.R., HADFIELD, M.G., ROSENBLUM, W.1. (1978). Association of Central Pontine Myelinolysis and Marchiafava-Bignami Disease. Neurology (Minneap.) 28: 1295-1298.

GOEBEL, H.D., ZUR, P.H.B. (1972). Central Pontine Myelinolysis. Brain 94: 495-504.

JANETT, D.L. (1970). Volume Conducted Potentials in Response to Auditory Stimuli as Detected by Aneraging in the CatElectroencephalogr. Clin. Neurophysiol. 20: 607-610. 
JEWETT. D.L.. ROMANO, M.N.. WILLISTON. J.S. (1970). Human Auditory Evoked Responses. Possible Brainstem Components Detected on the Scalp. Science 167: 15171518.

MESSERT, B., ORRISON, W.W., HAWKINS. M.J. et al (1979). Central Pontine Myelinolysis. Neurology (Minneap.) 29: 147-160.

OCHS, R., MARKAND, O.N., DEMYER, W.E. (1979). Brainstem Auditory Evoked Responses in Leucodystrophies. Neurology (Minneap.) 29: 1089-1093.

PAGUIRIGAN, A., LETKEN, E.G. (1969). Central Pontine Myelinolysis. Neurology (Minneap.) 19: 1007-1011.

POINERS, J.M.. McKEENER, P.E. (1976). Central Pontine Myelinolysis: An Ultrastructural and Elemental Study. J.
Neurol. Sci. 29: 65-81.

ROBINSON, K., RUDGE, P. (1977). Abnormalities of the Auditory Evoked Potentials in Patients with Multiple Sclerosis. Brain 100: 19-40.

SATYA-MURTI, S.. CACACE, A.T., HANSON, P.A. (1978). Abnormal Auditory Evoked Responses in Hereditary MotorSensory Neuropathy. Ann. Neurol. 5: 445478.

STARR, A. (1976). Auditory Brainstem Responses in Brain Death. Brain 99: 543554

STARR, A., ACHOR, L.J. (1975). Auditory Brainstem Responses in Neurological Disease. Arch. Neurol. 32: 761-768.

STOCKARD, J.J., ROSSITER, V.S. (1977) Clinical and Pathological Correlates of
Brainstem Auditory Evoked Response Abnormalities. Neurology 27: 316-325.

STOCKARD, J.J., ROSSITER, V.S., WIEDERHOLT, W.C. et al (1976). Brainstem Auditory Evoked Responses in Suspected Central Pontine Myelinolysis. Arch. Neurol. 33: 726-728.

TELFER, R.B., MILLER, E.M. (1979). Central Pontine Myelinolysis Following Hyponatremia Demonstrated by Computerized Tomography. Ann. Neurol. 6: 455-456.

TOLOSA, E.S., ZEESE, J.A. (1979). Brainstem Auditory Evoked Responses in Progressive Supranuclear Palsy. Ann. Neurol. 6: 369.

WIEDERHOLT, W.C., KOBAYASHI, R.M., STOCKARD, J.J. et al (1977). Central Pontine Myelinolysis. Arch. Neurol. 34: 220223. 